\title{
Fabrication and lasing characteristics of GaN nanopillars
}

\author{
Ming-Hua Lo ${ }^{1,2}$, Yuh-Jen Cheng ${ }^{1,2}$, Hao-Chung Kuo ${ }^{1}$, Shing-Chung Wang ${ }^{1}$ \\ ${ }^{1}$ Department of Photonics and Institute of Electro-Optical Engineering, \\ National Chiao Tung University, 1001 Ta Hsueh Rd., Hsinchu 300, Taiwan \\ ${ }^{2}$ Research Center for Applied Sciences, Academia Sinica, Taipei 11529, Taiwan
}

\begin{abstract}
We report the fabrication of $\mathrm{GaN}$ nanopillars and their laser action characteristics under optical pumping measurement. The nanopillars were fabricated from a GaN epitaxial wafer by self-assembled Ni nanomasked etching, followed by epitaxial regrowth to form crystalline facets on the etched nanopillars. The regrowth process is intended to reduce surface defects created during ICP-RIE etching. The density of etched GaN nanopillars is about $8.5 \times 10^{8} / \mathrm{cm}^{2}$ and the diameter and height of $\mathrm{GaN}$ nanopillars are about $250 \mathrm{~nm}$ and $650 \mathrm{~nm}$, respectively. The as grown GaN nanopillars exhibit a random distribution with hexagonal pillar geometry. The sample is optically excited by frequency tripled Nd:YAG pulsed laser. The Gaussian waist of pumping spot is $1.8 \mathrm{um}$. At low pumping intensity, the emission has a broad spontaneous emission spectrum with maximum at $363 \mathrm{~nm}$. As pump intensity increases, a narrow peak at $363 \mathrm{~nm}$ emerges quickly from the broad spontaneous emission back ground. The lasing action occurs at threshold pump power density of $122 \mathrm{MW} / \mathrm{cm}^{2}$. The emission linewidth decreases with pumping power across threshold and reaches a lowest value of about $0.38 \mathrm{~nm}$ above threshold. The excitation-power-dependent spectra show that the lasing wavelength has a slight blue shift as pump power increases. We remark that this is due the band filling of the increasing excited carrier density.
\end{abstract}

Keywords: GaN nanopillar, GaN laser, excitonic emission.

\section{INTRODUCTION}

$\mathrm{GaN}$ is considered as a very promising material for UV and blue optoelectronic devices due to its wide direct bandgap transition and strong emission properties. GaN one-dimensional (1-D) nano structure devices have attracted great research interests for their novel properties and potential applications [1,2]. These nano devices, known as nanopillars or nanorwires, can be grown by selective area or self assembled growth using rf-plasma-assisted molecular beam epitaxy (rf-MBE) [3] or metal organic chemical vapor deposition (MOCVD) [4]. The selective area growth can produce arrays of nanopillars in designed order while self assembled growth often produces nanowires in random orientation. The fabricated nanowires or nanopillars have shown improved material quality and size confinement effect which can significantly enhance light emitting properties [5-7].

The optical properties of GaN nanowires are usually investigated by photoluminescent (PL) measurement. The reported room temperature stimulated emission peaks are often at wavelengths longer [8-10] than the intrinsic GaN excitonic transition $363 \mathrm{~nm}[11,12]$. The redshift of stimulated emission peak has also been observed in intensively pumped GaN thin film material [13-18]. The redshift can be due to several different mechanisms. At carrier density above Mott transition, the redshift is attributed to the bandgap shrinkage due to many body interaction [15], where excitons are disassociated into electron-hole plasma [10,14]. Other mechanisms involve exciton-exciton or exciton-phonon scattering but occur only at cryogenic temperature. A room temperature stimulated emission directly from excitonic transition in the as grown $\mathrm{GaN}$ material has not been observed so far.

Here we report the observation of room temperature excitonic lasing action from optically pumped GaN nanopillars. The nanopillars were fabricated from a GaN epitaxy wafer by patterned etching and crystalline regrowth. The fabricated nanopillars have hexagonal crystalline facets with average diameter of $250 \mathrm{~nm}$ and length of $\sim 660 \mathrm{~nm}$ standing on a GaN substrate. When the sample was pumped by a focused $355 \mathrm{~nm}$ laser with $1.8 \mu \mathrm{m}$ spot size, a narrow excitonic lasing peak emerged from broad spontaneous emission background. The lasing wavelength maintains at $\mathrm{GaN}$ exciton transition wavelength through out the observed pump power range. The carrier density near the threshold of lasing action is estimated to be above typical Mott transition density of GaN. It is very interesting to observe the persistence of exciton

Gallium Nitride Materials and Devices VI, edited by Jen-Inn Chyi, Yasushi Nanishi,

Hadis Morkoc, Joachim Piprek, Euijoon Yoon, Proc. of SPIE Vol. 7939, 79391T · @ 2011

SPIE - CCC code: $0277-786 X / 11 / \$ 18 \cdot$ doi: $10.1117 / 12.874151$

Proc. of SPIE Vol. 7939 79391T-1 
stimulated emission rather than the often reported redshifted electron-hole plasma emission at such high pump intensity. We attribute this lasing to the existence of excitons in short delay time after pump pulse $[19,20]$ and the strong exciton and photon interaction enabled by crystalline nanopillar structure.

\section{FABRICATION OF GAN NANOPILLARS}

The schematic of GaN nanopillar fabrication process is shown in Fig. 1(a)-(d). A $3 \mu \mathrm{m} \mathrm{GaN} \mathrm{thin} \mathrm{film} \mathrm{was} \mathrm{grown} \mathrm{on} \mathrm{a} \mathrm{c-}$ plane sapphire (0001) substrate by low pressure metalorganic chemical vapor deposition (MOCVD). A $300 \mathrm{~nm}$ Si3N4 thin film was subsequently deposited on the GaN thin film by plasma-enhanced chemical-vapor deposition, followed by the electron-beam evaporation of a $100 \mathrm{~nm}$ Ni thin film, as illustrated in Fig. 1 (a). The sample was then subjected to rapid thermal annealing (RTA) at $850{ }^{\circ} \mathrm{C}$ under nitrogen ambiance for $1 \mathrm{~min}$. to form self-assembled Ni nanomasks on Si3N4 film surface, as shown in Fig. 1(b). A reactive ion etching was conducted to etch Si3N4 film using a CF4/O2 gasmixture to transfer Ni nanomask pattern down to Si3N4 layer. The sample was subsequently etched down to GaN by inductively coupled plasma reactive ion etching system (SAMCO ICP-RIE 101iPH) operated at $13.5 \mathrm{MHz}$ under a gas mixture of $\mathrm{Cl} 2 / \mathrm{Ar}=50 / 20 \mathrm{sccm}$ for 2 min. to form nanopillars as shown in Fig. 1(c). The ICP source power and bias power of the ICP-RIE system were set at 400 and $100 \mathrm{~W}$, respectively. The sample was dipped into a nitric acid solution (HNO3) at $100{ }^{\circ} \mathrm{C}$ for $5 \mathrm{~min}$. to remove the Ni nano masks. Finally, the etched GaN sample was put back to MOCVD to grow additional $\mathrm{GaN}$ on the etched GaN nanopillars, as illustrated in Fig. 1(d). The Si3N4 mask was intentionally left on top of nanopillars to prevent the regrowth in c-axis direction. This regrowth process grew crystalline $\mathrm{GaN}$ on the etched pillar side walls. It reduces surface defects created during the above ICP-RIE pillar etching process.

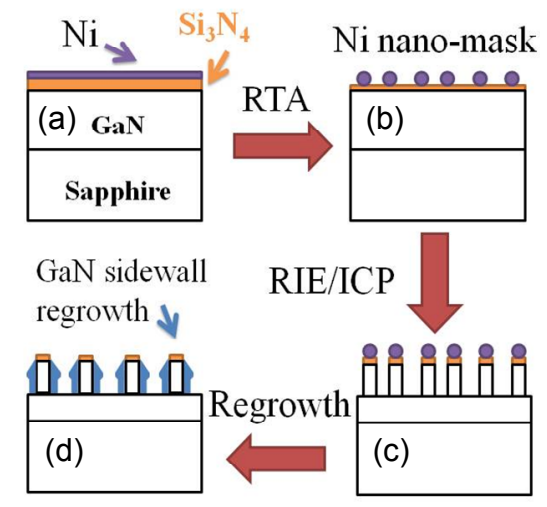

Fig. 1. (a)-(d) Nanopillar fabricating process flow: (a) Si3N4/Ni deposition. (b) Rapid thermal annealing (RTA) to form Ni nanomasks. (c) Ni nanomask pattern transferred to Si3N4 and GaN by dry etching. (d) Crystalline regrowth to from hexagonal pillars.

The SEM images of the fabricated nanopillars are shown in Fig. 2. Figure 2 (a) is the top view of the etched nanopillar sample before regrowth. The irregular shape was originated from the self-assembled Ni nanomasks. Figures 2 (b)-(c) are the top and side views of the nanopillars after regrowth. The regrowth grows hexagonal M-plane $\{11-20\}$ crystalline sidewall facets. The irregular hexagonal shape is due to the irregular etched nanopillar pattern as shown in Fig. 2 (a). The regrowth also grows inclined R-plane facets $\{11-22\}$ close to the top of nanopillars with a very slight overgrowth on Si3N4 mask. The height of nano-pillars is about $660 \mathrm{~nm}$. The averaged diameter is about $250 \mathrm{~nm}$. 

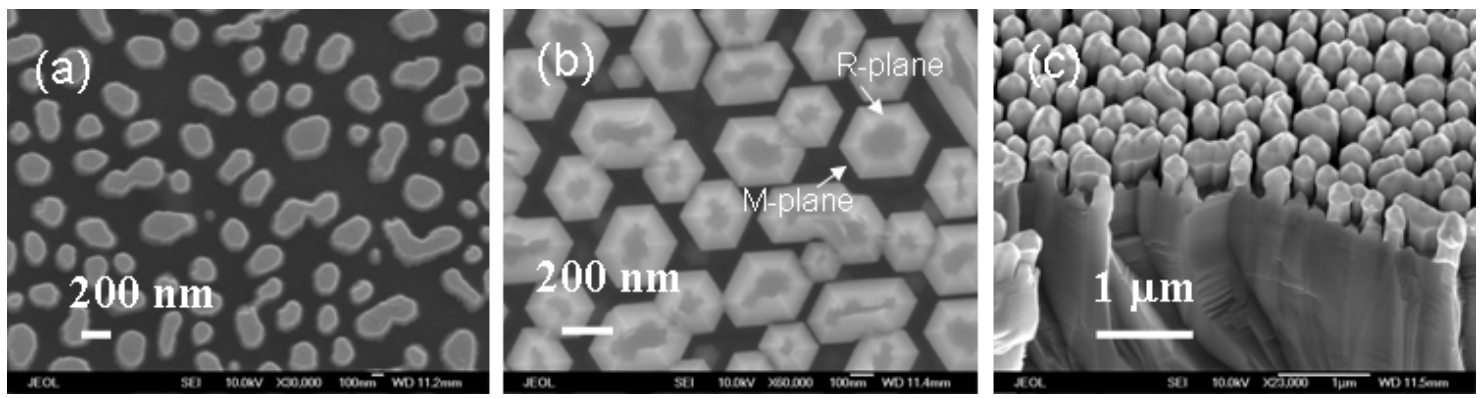

Figure 2. (a) Top view of the etched nanopillars before regrowth. (b) Top view of nanopillars after regrowth, showing the M-plane $\{11-20\}$ hexagonal side walls and the top inclined R-plane $\{11-22\}$ facets. (c) Side view of nanopillars after regrowth.

\section{OBSERVATION OF LASING ACTION}

The optical property of the fabricated GaN nanopillars was probed by time-averaged PL measurement using tripled $\mathrm{Nd}$ :YAG pulse laser at $355 \mathrm{~nm}$. The pulse width is $0.5 \mathrm{~ns}$ and the pulse repetition rate is $1 \mathrm{KHz}$. The laser beam was focused on sample surface in normal incidence by a 15X UV microscope objective. The focus spot had a Gaussian beam waist of $1.8 \mu \mathrm{m}$, which is verified by knife edge measurement. The PL signal was collected through the same $15 \mathrm{X}$ objective and analyzed by a spectrometer (Jobin Yvon IHR320). At low pump intensity, the spontaneous emission has a maximum around $363 \mathrm{~nm}$, as shown in Fig. 3 (a). This wavelength is the same as the typical exciton transition reported in GaN thin film [11,12]. To verify that this is the exciton emission of nanopillar, a collection of nanopillars were scraped off from the sample on a quartz template for transmission loss spectrum measurement. Another blank quartz template was used as a reference to calibrate out the loss due to quartz template. The spectral difference between these two samples reveals an absorption peak at $363 \mathrm{~nm}$ together with band to band transition absorption at slightly shorter wavelength. This spectral characteristic is identical to the typical measured $\mathrm{GaN}$ thin film absorption spectrum (see Fig. 1 (a) in Ref. [12]), where the absorption peak is a signature of excitonic transition. The same spectral characteristic was also observed in the GaN thin film substrate used to fabricate nanopillars by ellipsometer measurement. The observed $363 \mathrm{~nm}$ spontaneous emission of nanopillars is therefore verified to be exciton emission. It is expected that the exciton emission wavelength is not altered by the nanostructure because the nanopillar diameter $250 \mathrm{~nm}$ is much larger than GaN exciton Bohr radius 2-3 nm. As pump intensity increases, a small peak emerges from the center of spontaneous emission, shown Fig. 3 (a). It is interesting to see its dramatic increase on top of the broad spontaneous emission background when pump intensity continues to increase as shown in Fig. 3 (b). The emergence of the narrow peak with much faster increasing rate than the spontaneous emission background indicates an onset of stimulated emission. We remark that the emerging peak is an excitonic lasing action.
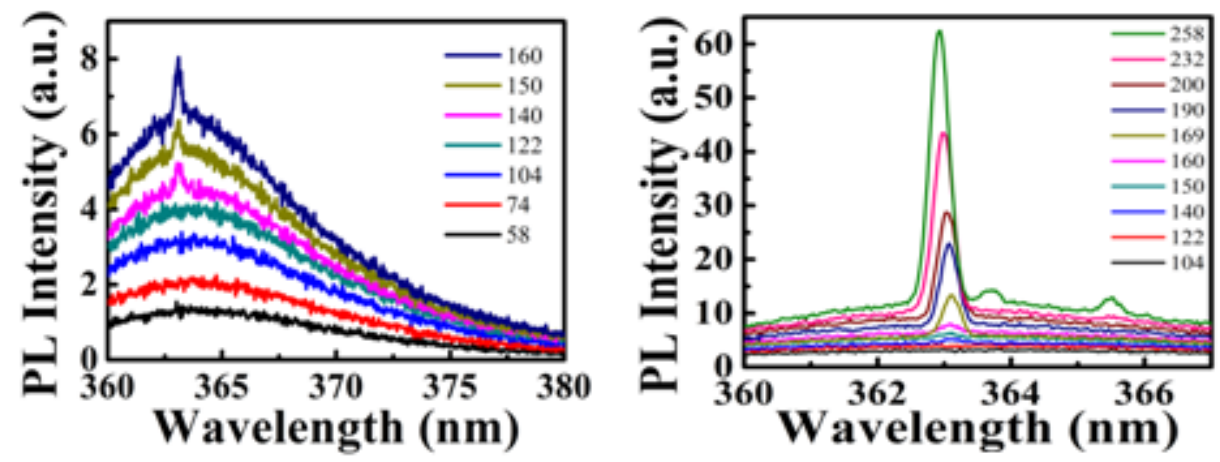

Figure 3. (a) Spontaneous PL spectra at various pump intensities below and near threshold. (b) PL spectra at various pump intensity levels showing the onset of $363 \mathrm{~nm}$ excitonic lasing action. Legend: pump intensity (MW/cm2). 
To better reveal the lasing behavior, the emerging peak intensity and the spontaneous emission intensity versus pump intensity are show in Fig. 4 (a). The spontaneous emission intensity is evaluated at wavelength close to the emerging peak. The increasing rate of the $363 \mathrm{~nm}$ emission peak (red line) is 9 times faster than that of spontaneous emission (black line). This threshold behavior of $363 \mathrm{~nm}$ emission clearly demonstrates the onset of lasing action. The linewidth versus pump intensity is also shown in Fig. 4 (a). The linewidth significantly reduces across the threshold down to 0.3 $\mathrm{nm}$. The stimulated emission wavelength versus pump intensity is shown in Fig. 4 (b). The wavelength slightly blue shifts from $363.1 \mathrm{~nm}$ to $362.9 \mathrm{~nm}$ when pump intensity increases from threshold to 1.7 times above threshold. We remark that this slight blueshift of emission wavelength with increasing pump intensity is due to excitonic band filling. This is in drastic contrast to the often reported redshifting stimulated emission with increasing pump intensity in GaN nanowires [8]. The redshift is often attributed to bandgap renormalization at carrier density above Mott transition [14]. To estimate the excited carrier density, we measured the reflected $355 \mathrm{~nm}$ power from a GaN nanopillar sample using a large area detector with edge filter to block $\mathrm{GaN}$ spontaneous emission. The pump laser was in normal incident to the sample through a 50/50 reflector. The detector was positioned from normal to oblique collection angles. The reflective power was mainly in normal direction and negligible in oblique direction. The measured total reflective power was only $0.3 \%$. There was no measureable transmitted power. From the calibration, the pump pulse intensity absorbed by nanopillars at lasing threshold is $200 \mathrm{MW} / \mathrm{cm}^{-2}$. Given the absorption coefficient of $1 \times 10^{5} \mathrm{~cm}^{-1}$ and carrier recombination coefficient $1.3 \times 10-8 \mathrm{~cm} 3 \mathrm{~s}-1$ from reported studies [21,22], the excited carrier density at threshold pump intensity is $5.2 \times 10^{19} \mathrm{~cm}^{-3}$. This is much higher than the typical reported Mott transition density $1.8 \times 10^{18} \mathrm{~cm}^{-3}[11,14]$. Even though the carrier density is significantly above Mott density, the observed stimulated emission still occurs at exciton wavelength in our nanopillar sample. It has been observed in a time resolved pump probe measurement that excitons can exist above Mott transition density in a short delay time after pump pulse [19,20]. We remark that this existence of exciton and the increased exciton-photon interaction due to photons scattered among nanopillars makes excitonic stimulated emission possible before excitons are disassociated into electron-hole plasma at above Mott transition density.
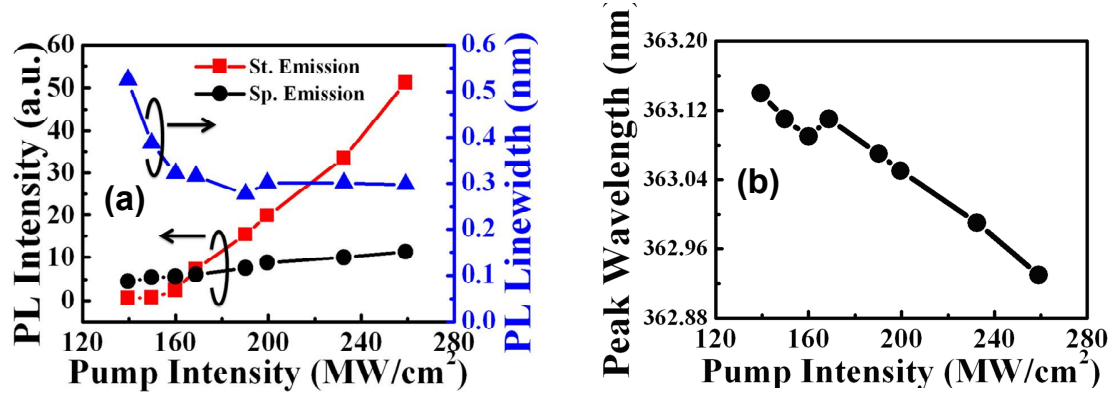

Figure 4. (a) The $363 \mathrm{~nm}$ stimulated (st.) emission intensity, linewidth, and spontaneous (sp.) emission intensity versus pump intensity. (b) The blueshift of stimulated emission wavelength with increasing pump intensity.

It is worth to note that the lasing linewidth is only about $0.3 \mathrm{~nm}$, significantly smaller than the most reported values from $\mathrm{GaN}$ nanowires [8-10]. For nanowires, the lasing modes are often the Fabry-Perot modes defined by the reflection from two nanowire end surfaces. There are often multiple lasing peaks because of the long wire length. The nanopillars in our experiment are short columns only about $660 \mathrm{~nm}$ standing on a GaN substrate. It is unlikely to have lasing modes oscillating in the pillar axis direction since there is only one reflection interface at one end. It is also unlikely due to the whispering gallery modes defined by light reflected among hexagonal crystalline sidewalls. The pump spot area covers a few nanopillars and the size of nanopillars has a distribution. Different nanopillars will have different whispering gallery mode resonances. If the lasing action is due to whispering gallery mode resonance, there would be multiple peaks. We remark that crystalline nanopillars provide better exciton confinement and also allow photons to scatter among them several times before escaping from the pumped area. As a result, photons have more chance to interact with excitons. It is very unique that such a strong exciton-photon interaction enables the lasing action with rather narrow linewidth even though there is no well defined conventional cavity. This is in contrast to bulk GaN material where there is no lateral confinement to enhance exciton and photon interaction. This may explain why direct excitonic lasing action has not been observed in bulk GaN material. 


\section{SUMMARY}

We report a room temperature excitonic lasing from optically pumped GaN nanopillars. The nanopillars were fabricated by self-assembled Ni nanomasked etching of an epitaxial GaN substrate, followed by crystalline regrowth to form hexagonal crystalline facets. When the sample was excited by a $355 \mathrm{~nm}$ laser with $1.8 \mu \mathrm{m}$ focused spot size, a lasing action was observed at $\mathrm{GaN}$ exciton transition wavelength. The excited carrier density at threshold pump power is significantly higher than Mott transition density. However, the lasing wavelength persists at exciton wavelength through out the observed pump power range. This is very different from the often reported redshifted lasing wavelength, where electron-hole plasma is the dominant gain mechanism at carrier density above Mott transition. We remark that the excitonic lasing is due to the persistence of excitons in short delay time after pump and the stronger exciton and photon interaction in the fabricated crystalline nanopillars, which induces the excitonic stimulated emission before excitons are disassociated into electron-hole plasma.

\section{ACKNOWLEDGEMENTS}

We thank the National Science Council of Republic of China (ROC) Taiwan for the support of this research under contract NSC97-2112-M-001-027-MY3, and Academia Sinica Nano-program.

\section{REFERENCES}

1. T. Kuykendall, P. Pauzauskie, S.K. Lee, Y.F. Zhang, J. Goldberger, and P. Yang, "Metalorganic chemical vapor deposition route to GaN nanowires with triangular cross sections," Nano Lett. 3, 1063-1066 (2003).

2. F. Qian, S. Gradečak, Y. Li, C.-Y. Wen, and C. M. Lieber, "Core/Multishell nanowire heterostructures as multicolor high-efficiency light-emitting diodes," Nano Lett. 5, 2278-2291 (2005).

3. K. Kishino, H. Sekiguchi, and A. Kikuchi, "Improved Ti-mask selective-area growth (SAG) by rf-plasmaassisted molecular beam epitaxy demonstrating extremely uniform GaN nanocolumn arrays," J. Cryst. Growth 311, 2063-2068 (2009).

4. S. D. Hersee, X. Sun, and X. Wang, "The controlled growth of GaN nanowires," Nano Lett. 6, 1808-1811 (2006).

5. N. Thillosen, K. Sebald, H. Hardtdegen, R. Meijers, R. Calarco, S. Montanari, N. Kaluza, J. Gutowski, and H. Lüth, "The state of strain in single GaN nanocolumns as derived from micro-photoluminescence measurements," Nano Lett. 6, 704-708 (2006).

6. E. Calleja, M. A. S. Garcia, F. J. Sanchez, F. Calle, F. B. Naranjo, E. Munoz, U. Jahn, and K. Ploog, Luminescence properties and defects in GaN nanocolumns grown by molecular beam epitaxy," Phys. Rev. B 62, 16826 (2000).

7. T. Kouno, K. Kishino, K. Yamano, and A. Kikuchi, "Two-dimensional light confinement in periodic InGaN/GaN nanocolumn arrays and optically pumped blue stimulated emission," Opt. Express 17, 20440-20447 (2009).

8. J. C. Johnson, H.-J. Choi, K. P. Knutsen, R. D. Schaller, P. D. Yang, and R. J. Saykally, "Single gallium nitride nanowire lasers," Nature mater. 1, 106-110 (2002).

9. H.-J.; Choi, J. C. Johnson, R. He, S.-K. Lee, F. Kim, P. Pauzauskie, J. Goldberger, R. J. Saykally, P. Yang, "SelfOrganized GaN Quantum Wire UV Lasers,” J. Phys. Chem. B, 107, 8721-8725 (2003).

10. S. Gradečak, F. Qian, Y. Li, H.-G. Park, and C. M. Liebera, "GaN nanowire lasers with low lasing thresholds," Appl. Phys. Lett. 87, 173111-3 (2005).

11. S. Shokhovets, K. Köhler, O. Ambacher, and G. Gobsch, "Observation of Fermi-edge excitons and exciton-phonon complexes in the optical response of heavily doped n-type wurtzite GaN," Phys. Rev. B 79, 045201-11 (2009).

12. A. J. Fischer, W. Shan, J. J. Song, Y. C. Chang, R. Horning, and B. Goldenberg, "Temperature-dependent absorption measurements of excitons in GaN epilayers," Appl. Phys. Lett. 71, 1981-1983 (1997).

13. O. Gluschenkov, J. M. Myoung, K. H. Shim, and K. Kimb, Z. G. Figen, J. Gao, and J. G. Eden, "Stimulated emission at $300 \mathrm{~K}$ from photopumped GaN grown by plasma-assisted molecular beam epitaxy with an inductively coupled plasma source, Appl. Phys. Lett. 70, 811-813 (1997).

14. F. Binet, J. Y. Duboz, J. Off, and F. Scholz, "High-excitation photoluminescence in GaN: Hot-carrier effects and the Mott transition," Phys. Rev. B 60, 4715-4722 (1999).

15. S. Bidnyk, T. J. Schmidt, B. D. Little, and J. J. Song, "Near-threshold gain mechanisms in GaN thin films in the temperature range of 20-700 K," App. Phys. Lett. 74, 1-3 (1999). 
16. K. Kazlauskas, G. Tamulaitis, A. Žukauskas, T. Suski, P. Perlin, M. Leszczynski, P. Prystawko, and I. Grzegory, "Stimulated emission due to spatially separated electron-hole plasma and exciton system in homoepitaxial GaN," Phys. Rev. B 69, 245316-9 (2004).

17. X. Zhang, P. Kung, A. Saxler, D. Walker, M. Razeghi, "Observation of room temperature surface-emitting stimulated emission from GaN:Ge by optical pumping," J. Appl. Phys. 80, 6544-6546 (1996).

18. Y. C. Chang, Y.-L. Li, D. B. Thomson, and R. F. Davis, "Phonon-assisted stimulated emission from pendeoepitaxy GaN stripes grown on 6H-SiC substrates," Appl. Phys. 91, 051119-3 (2007).

19. Y.-C. Huang, G.-W. Chern, K.-H. Lin, J. C. Liang, C. K. Sun, C. C. Hsu, S. Keller, and S. P. DenBaars, "Femtosecond dynamics of exciton bleaching in bulk GaN at room temperature," Appl. Phys. Let. 81, 85-87 (2002).

20. C. K. Choi, Y. H. Kwon, J. S. Krasinski, G. H. Park, G. Setlur, J. J. Song, and Y. C. Chang, "Ultrafast carrier dynamics in a highly excited GaN epilayer," Phys. Rev. B 63, 115315-6 (2001).

21. J. F Muth, J. H. Lee, I. K. Smagin, R. M. Kolbas, H. C. Casey, B. P. Keller, U. K. Mishra, and S. P. DenBaars, "Absorption coefficient, energy gap, exciton binding energy and recombination lifetime of GaN obtained from transmission measurements," Appl. Phys. Lett. 71, 2572-2574 (1997).

22. F. Binet, J. Y. Duboz, E. Rosencher, F. Scholz, and V. Harle, "Mechanisms of recombination in GaN photodetectors," Appl. Phys. Lett. 69, 1202-1204 (1996). 